\title{
Mechanistic Interpretations of Some Empirical Correlations in Creep and Creep Fracture
}

\author{
Geoffrey W. GREENWOOD
}

School of Materials, The University of Sheffield, Mappin Street, Sheffield, S1 3JD, U.K.

(Received on December 26, 1989; accepted in the final form on March 9, 1990)

\begin{abstract}
Creep failure occurs through excessive strain or premature fracture. From many empirical relationships proposed to predict the strain incurred in a given time at a specified stress and temperature, some are selected in terms of their practical usefulness and their scientific basis is discussed. It is shown that distinctive mechanisms can operate, dependent upon the material and on the creep conditions and it is important that these are individually considered. It is also noted that these mechanisms may be coupled in ways which may smooth the transitions between them. This may account for some empirical approaches being applicable for approximate predictions over relatively wide ranges. Different mechanisms leading to creep fracture can also be distinguished. Limits of extreme behaviour can be clearly identified and explained but, under intermediate conditions, the form of coupling of different mechanisms leads to continuity in transitional regions. Concepts of creep damage can be developed in terms of cavity nucleation and growth, with the value of microstructural examination clearly recognised. Whilst much remains to be done, a scientific framework for the understanding of the mechanisms of creep and creep fracture is already in place and there is opportunity for detailed physical modelling to assist the prediction of behaviour in practical situations.
\end{abstract}

KEY WORDS: creep; fracture; mechanisms; life prediction.

\section{Introduction}

In the simplest situation, the useful service life of a component operating at elevated temperatures may be estimated from a knowledge of the relationship of creep strain and time when the material is subjected to an appropriate range of stress and temperature and from the further knowledge of the condition under which fracture may occur.

Much data has been gathered in these areas, ${ }^{11}$ enough to have led to a multitude of empirical correlations that can readily be applied to the prediction of creep life. ${ }^{2,31}$ Sometimes such predictions have been reliable but this is not always the case. Large margins of error must still be accepted and it is a matter of serious concern to decide how these margins of error may be reduced.

A full scientific appraisal of the micromechanisms of the behaviour of materials at elevated temperatures ${ }^{4}$ could provide the most secure means of alleviating problems but difficulties should not be underestimated. At such temperatures environmental interactions may arise;

(1) Chemical changes may occur, with elements rearranging their affinity and location.

(2) Microstructure may alter as precipitates coarsen or change thcir form and as grain boundaries migrate or slide.

(3) Dislocations may multiply and annihilate and climb as well as glide.

(4) Vacancy generation, movement and absorption may also be directionally influenced by the con- ditions imposed.

Simultaneously, creep damage is likely to occur, influenced both by external forces and by internal microstructural and chemical features. Against this background, notwithstanding major scientific progress, it is clear that well founded predictions of creep life are still far from achieving the required accuracy for many practical situations.

Empirical correlations should therefore not be discarded but, wherever possible, be linked to some underlying mechanisms so that predictive methods can become more firmly based, with their range of applicability more realistically defined. ${ }^{5}$ ) Such an approach can most effectively lead to a physical modelling of the processes taking place. At this stage numerical methods of solution will become necessary for detailed treatment of specific circumstances.

The aim of the present paper is to examine a few of the features that govern the occurrence of creep strain and creep fracture so that some of the main problems related to the prediction of the time prior to the occurrence of high temperature failure become more clear.

\section{The Effects of Imposed Conditions}

Ignoring environmental influences, it is required to determine creep strain $\varepsilon$ as a function of stress $\sigma$, temperature $T$, time $t$ for a material whose structure is characterised by $s$, such that we may write

$$
\varepsilon=f(\sigma, T, t, s)
$$


For a creep lifetime $t=t_{f}$ we require $\varepsilon<\varepsilon_{\min }$ which is the limiting allowable value of strain. It is further necessary that fracture should not occur before this lifetime is achieved so that we have the additional requirement that the time before fracture $t_{R}>t_{f}$ where $t_{R}$ may be described by

$$
t_{R}=g(\sigma, \mathcal{T}, s)
$$

Formulated in this way, the problems of creep strain and fracture prediction have a deceptively simple appearance. In practice, the situation is highly complex. This is primarily because all the parameters are not independent and because the form of the functions $f$ and $g$ may be determined by the range of values of each of the parameters they contain. Even for the same material, the effect of $s$ is influenced by $\sigma$ and $T$, and $s$ may also change substantially with $t$. The changes in $s$ throughout creep life are subtle. Inevitably, similar types of processes take place that occur on long term heat treatment, but these may be modified by the imposed stress and resulting strain.

Additionally, creep damage accumulates in a way which depends on all the other parameters. It is this damage that can eventually cause quite sudden fracture in creep and so its characterisation is of vital importance. Thus the formulations in Eqs. (1) and (2) can obscure the variety of phenomena that are observable and play a significant part in creep life evaluation. It will be also noted that no attempt has been made here to evaluate the effects of temperature or stress variation or of multiaxial stress application that may again totally change the materials' behaviour from that under constant temperature and constant uniaxial stress conditions.

Despite this cautionary approach to the unjustifiable interpretation of Eqs. (1) and (2), it would nevertheless be unwise to discard them entirely for they, at least, encapsulate the nature of the problem, providing it is appreciated that the form of the functions $f$ and $g$ is influenced by the range of values of the parameters they contain and that the parameter $s$ characterising the material incorporates all the conditions and time dependent changes including the progressive creep damage.

As an example of the use of these equations we may consider first the effect of stress $\sigma$ on the strain $\varepsilon$ that is reached in a given time $t$ and next on the effect of stress on the time before fracture $t_{R}$ for any material at a constant temperature. Details of these relationships may vary considerably but their general form is illustrated schematically in Figs. 1 and 2. The shape of both these curves is such that any extrapolation made on the basis of a tangential construction from high stress or short term data is likely to indicate a better durability of the material than that which may be displayed in long term service. These features lie at the centre of the problem of creep life prediction and will be considered in more detail.

\section{The Stress Dependence of Greep Strain}

The relationship between $\varepsilon$ and $\sigma$ at constant time

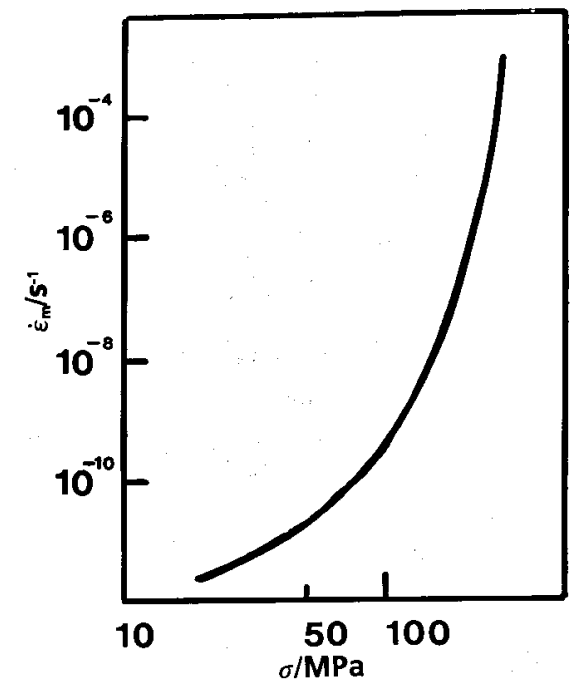

Fig. 1(a). The experimentally determined relationship between minimum creep rate $\dot{\varepsilon}_{m}$ and stress $\sigma$ for $0.5 \mathrm{Cr}-0.5 \mathrm{Mo}-0.25 \mathrm{~V}$ Steel at $565^{\circ} \mathrm{C}$.

Note that the extrapolation from high to low stress levels on the basis of a power law relation would lead to the prediction of creep rate lower than that observed.

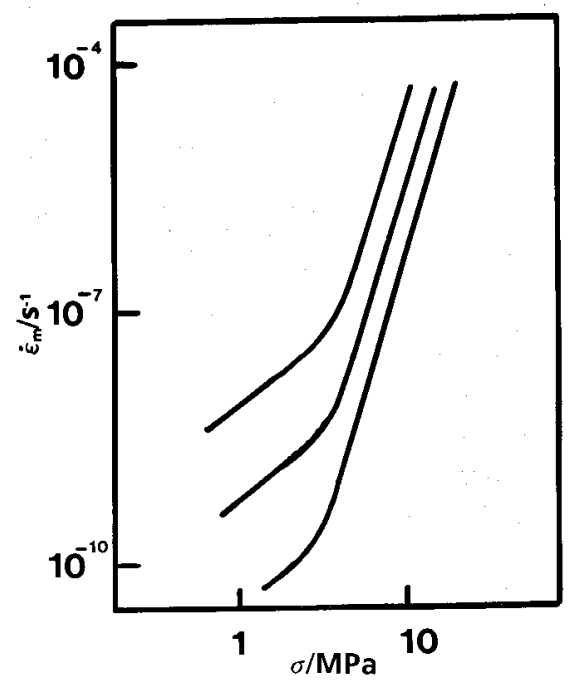

Fig. 1(b). The experimentally determined relationship between $\dot{\varepsilon}_{m}$ and $\sigma$ at $450^{\circ} \mathrm{C}$ for $\mathrm{Mg}, 0.8$ mass $\%$ $\mathrm{Al}, 0.005 \mathrm{mass} \% \mathrm{Be}$.

Here there is a more distinct transition between different mechanisms with behaviour dependant on grain size.

The grain size for the upper curve $20 \mu$, for the centre curve $80 \mu$ and for the lower curve $300 \mu$.

and temperature is influenced by the shape of the creep curve and so it depends on the creep conditions and on the particular material. Relations in the primary and tertiary creep stages may differ from each other and from that in the secondary stage. In many technological materials the secondary stage may be difficult to identify in so far as creep rate may not be constant over a significant period of time. ${ }^{6)}$ In such cases, it is appropriate to define a minimum creep rate $\dot{\varepsilon}_{m}$ with its importance justified by the observation that the creep rate is generally near its minimum value for 


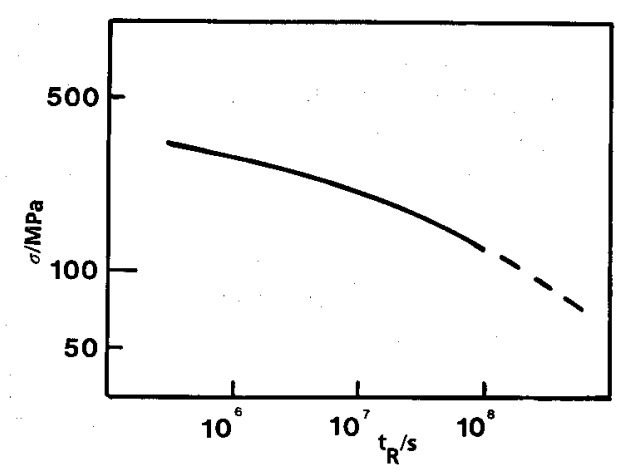

Fig. 2. The experimental relationship between stress $\sigma$ and time to fracture $t_{R}$ for a $0.5 \mathrm{Cr}-0.5 \mathrm{Mo}-0.25 \mathrm{~V}$ Steel at $565^{\circ} \mathrm{C}$. The dotted line indicates the uncertainty in extrapolating data beyond $10^{8} \mathrm{~s}$.

a substantial part of creep life. The dangers inherent in this approach are clear when progressive acceleration of creep rate becomes apparent in the tertiary stage. The effect of stress on creep strain during this stage requires much further exploration but it is doubtful whether widely applicable results will be readily forthcoming. Methods of mathematical modelling are likely to present the best way forward in analysing such results.

Despite the present cautious approach to the use of the minimum creep rate $\dot{\varepsilon}_{m}$, this still presents the most direct way of comparing the effect of stress on different materials. Much experimental information is available and its theoretical basis is beginning to be understood. ${ }^{4}$ In some instances a reasonably good fit of experimental results with $\dot{\varepsilon}_{m}$ proportional to exp $\sigma$ can be achieved and in most cases this relationship can satisfactorily describe the curve at the higher stress levels. At lower stresses a relationship of the form $\dot{\varepsilon}_{m} \propto \sigma^{n}$ is more appropriate but with $n$ tending to decrease from values of about 5 down to unity for pure metals. This decrease of $n$ with stress is a common factor of creep behaviour though with $n$ having a value of about 3 over an extensive stress range for solid solution alloys and with $n$ able to assume a value larger than 5 for strong particle hardened creep resisting alloys subjected to high stress.

There now seems substantial evidence that the overall relation between $\dot{\varepsilon}_{m}$ and $\sigma$ is determined by a variety of mechanisms, each operating over distinction ranges of stress and temperature but with these mechanisms coupled in a number of ways. Starting at the highest stress levels, much data suggests that

$$
\dot{\varepsilon}_{m}=\AA_{0} \exp -\left[\left\{1-\left(\sigma / \sigma_{0}\right)\right\} \Delta F / k T\right]
$$

where, $\Delta F$ : an activation barrier to dislocation movement

$\dot{\varepsilon}_{0}$ : a frequency factor incorporating the mobile dislocation density and sweep step of a dislocation segment after its activation $^{7}$

$\sigma_{0}:$ athermal resistance to deformation.

As the stress is lowered Eq. (3) can be linked ${ }^{8 \prime}$ in a smooth transition with a power law creep relation

$$
\dot{\varepsilon}_{m}=A(\sigma / G)^{n}
$$

where $G$ is the shear modules and $A$ is constant but is dependent on temperature and, to a lesser extent, is influenced by $n$. The most convincing theoretical analyses of dislocation creep lead to the expectancy for a value of $n=3$ when dislocation mobility is impeded by solute drag and this is the value generally revealed by experiments for solid solution alloys subjected to intermediate stresses and temperatures. ${ }^{4}$ ) The value of $n=5$ for pure metals under similar conditions may be interpreted in a generally similar way but with diffusion processes occurring primarily along dislocation cores with the dislocation density being determined by the square of the applied stress. ${ }^{8}$

The considerations just mentioned have usually predominated in discussions of creep mechanisms, yet, in practice, for alloys expected to remain in service for several decades, a lower stress sensitivity with $n=2$ and sometimes as low as unity is noted under stresses sufficiently low to match this long life requirement. ${ }^{9}$ It is in such circumstances that different creep mechanisms must be invoked. The proposal of a creep mechanism with $n=1$ was first put forward ${ }^{10)}$ about 40 years ago and was then considered to be essentially of academic rather than practical importance since it was believed to be significant only at temperatures close to the melting point. The recognition, ${ }^{11)}$ some 15 years later, of the likely importance of grain boundary diffusion to this mechanism, which involves the directional movement of vacancies, led to a realisation of its practical relevance for fine grained materials.

Apart from the need to make some simplifying assumptions about grain shapes, the rate of diffusional creep can be completely calculated with the contributions of lattice and grain boundary diffusion being additive leading to the formula

$$
\dot{\varepsilon}=\frac{14 D_{\sigma} \Omega}{k T d^{2}} \cdot\left[1+\frac{\pi D_{g} w}{D d}\right]
$$

where, $\dot{\varepsilon}:$ the creep rate under stress $\sigma$ at a temperature $\mathcal{T}$ in material of grain size $d$ and atomic volume $\Omega$

$D, D_{g}$ : the lattice and the grain boundary self diffusion coefficient with $w$ the grain boundary width, respectively

$k$ : Boltzmann's constant.

Eq. (5) has generally been well supported in experiments on pure metals at low stresses but marked deviations have generally been found for creep resisting alloys. Thus diffusional creep theory in its original form does not represent a lower limit to creep strength.

For many alloys it seems that below a certain threshold stress, grain boundaries are incapable of emitting vacancies and that, even above this stress, the rate of vacancy emission may be slower than the rate of vacancy diffusion between different grain boundaries. Models based on the emission and absorption of vacancies at grain boundary dislocations seem to offer ${ }^{12)}$ the best hope of understanding the 
emission and absorption process and lead to the prediction of a creep rate $\dot{\varepsilon} \propto \sigma^{2} / d$.

From the above discussion it is clear that a range of response to applied stress is to be anticipated in the creep rate that is induced, which progressively increases in its sensitivity as the stress becomes higher. No single mathematical expression is likely to represent exactly the form of variation of $\dot{\varepsilon}$ with $\sigma$ over a wide range but approximate expressions may be reasonably representative over more limited ranges.

Sometimes it is possible to detect a relatively clear demarcation as one mechanism gives way to another as the stress is increased but, more often, transitions are smooth and mechanistic changes occur gradually.

It is important to appreciate the ways in which different mechanisms can be coupled in series or parallel operation together with a clear recognition of their individual characteristics. These characteristics are markedly sensitive to the composition and microstructure of the material and so it is immediately apparent that no single formulation can be totally adequate. The clearest example is in the contrast in parameters controlling dislocation and diffusional creep. The range of predominance of the latter is substantially extended by decrease in grain size. Moreover, the effects of grain shape anisotropy and of multiaxial stress application can then also be of major influence in diffusional creep. ${ }^{13,14}$ )

\section{Creep Strain Time Dependence}

The shape of the creep curve has provided many challenges for mathematical description ${ }^{6)}$ and its dependencies on stress and on temperature previously mentioned can have a major effect on its form. Much debate has recently ranged on the significance of the secondary creep stage with the proposal that it is often only of very short duration in creep resisting materials. Furthermore, formulation of the primary and tertiary stages and the addition of the terms describing each of these may lead to a nearly constant creep rate over a period which matches this duration. Such considerations have resulted ${ }^{15)}$ in the proposal that

$$
\varepsilon=\theta_{1}\left[1-\exp \left(-\theta_{2} t\right)\right]+\theta_{3}\left[\exp \left(\theta_{4} t\right)-1\right]
$$

where the four $\theta$ parameters are each functions of stress and temperature, suggested to take the form

$$
\log \theta_{i}=a_{i}+b_{i} T+c_{i} \sigma+d_{i} \sigma T
$$

Chemical and microstructural features are then incorporated in these four further parameters in $\mathrm{Eq}$. (7).

On such a basis it is apparent the sixteen parameters in total are considered sufficient for a full description of creep. This indicates the magnitude of the task of determining the minimum information for an assessment of the creep strain, without the requirement to identify individual creep mechanisms and specific creep regimes. With great effort required to obtain such information, it is clearly important to establish the reliability of the form of Eqs. (6) and
(7) and much attention ${ }^{6)}$ has been given to this aspect for it represents a method of extrapolation that can easily be adapted to the life prediction of engineering components provided sufficient data are available.

Some of the parameters can be incorporated on a mechanistic basis but some relationships take on an unfamiliar form. For example, the minimum creep rate $\dot{\varepsilon}_{m}$ can be obtained by differentiation of Eq. (6) to determine the point of inflexion. From this, it is deduced that

$$
\dot{\varepsilon}_{m}=\theta_{1} \theta_{2}\left(\theta_{3} \theta_{4}^{2} / \theta_{1} \theta_{2}^{2}\right)^{\theta_{2} /\left(\theta_{2}+\theta_{4}\right)}+\theta_{3} \theta_{4}\left(\theta_{1} \theta_{2}^{2} / \theta_{3} \theta_{4}^{2}\right)^{\theta_{4} /\left(\theta_{2}+\theta_{4}\right)}
$$

It follows from Eq. (7) that, at constant temperature each of the $\theta$ parameters may be written in the form

$$
\theta_{i}=A_{i} \exp \left(B_{i} \sigma\right)
$$

where, $A_{i}, B_{i}$ : new parameters related to $a_{i}, b_{i}, c_{i}$, $d_{i}$, and temperature.

Thus from Eqs. (8) and (9) the form of the $\dot{\varepsilon}_{m} v s . \sigma$ relationships may be derived.

There are further implications from these equations, one of which is the expectation of relationships between $\sigma$ and $T$ for the circumstances for which identical creep curves are produced. For each of the $\theta$ parameters, these relationships must take the form

$$
\sigma=\left(K_{i}-b_{i} T\right) /\left(c_{i}+d_{i} T\right)
$$

where $K_{i}=\log \theta_{i}-a_{i}$ and so $K_{i}$ is constant for any given value of $\theta_{i}$. These considerations demonstrate the scope of data collection and analysis that is required. This presents a formidable task and illustrates the importance of providing a scientific basis for correlation between parameters with a continuing search for more accurate formulations.

\section{Creep Fracture}

\subsection{Some Empirical Relationships}

The most frequently quoted relationship is that first proposed by Monkman and Grant ${ }^{16)}$ which relates the time to fracture $t_{R}$ and the minimum creep rate $\dot{\varepsilon}_{m}$ for many materials over extensive ranges of stress and temperature in the form

$$
t_{R} \propto 1 / \dot{\varepsilon}_{m}
$$

The simplest interpretation of Eq. (11) is that the fracture mode is uniquely controlled by the mechanism of deformation. Whilst this is often true for ductile fracture its wider applicability poses additional questions.

More generally, relationships have been sought between $t_{R}$ and $\sigma$ to determine the definitive expression of Eq. (2). This often takes the form

$$
t_{R} \propto 1 / \sigma^{m}
$$

Such an expression clearly has an important value in the prediction of creep life but much accumulation of data has indicated a decrease in the exponent $m$ with reduced stress making predictions on the basis of 
short term tests too optimistic. This is a central problem in the assessment of creep life and makes it necessary to obtain detailed information of the processes that lead to fracture at elevated temperatures.

Observations of creep fracture surfaces enable forms of creep damage to be assessed, with cracks tending to predominate at the higher stress levels and with cavities more important at lower stresses.

\subsection{Wedge Cracking}

This form of cracking arises at triple point junctions where three grain boundaries intersect. It originates from the stress induced grain boundary sliding and with a sliding rate $\dot{\varepsilon}_{s}$ in a material of grain size $d$ it has been deduced ${ }^{17)}$ that

$$
t_{R} \sim 2 \gamma / \dot{\varepsilon}_{s} \sigma d
$$

with $\gamma$ being the energy required to separate unit area of surface.

If $\dot{\varepsilon}_{*}$ can be approximately equated with $\dot{\varepsilon}_{m}$ and creep life is mainly influenced by the secondary creep stage, then with $\sigma$ proportional to $\dot{\varepsilon}_{m}^{1 / n}$ from Eq. (4), we anticipate

$$
t_{R} \propto 1 / \dot{\varepsilon}_{m}^{(n+1) / n}
$$

It is immediately clear that this approaches the Monkman-Grant relationship as the value of $n$ increases.

It is also noted from Eq. (13) that the time before creep fracture is inversely relation to grain size. Although this relationship is not always closely supported experimentally, it is nevertheless generally true that fine grained materials have a longer creep life and that a refinement in grain size and limitation of grain growth in welding form the best basis for extending creep life.

Although Eq. (14) is most easily explained on the basis of wedge cracking, it often remains approximately valid when failure occurs through the nucleation and growth of grain boundary cavities.

\subsection{Cavitation}

Cavitation is the major cause of creep failure in materials after long creep lives. Mechanisms for early growth have now become well established. Growth can occur through plastic deformation processes including creep and these predominate at rather high flow rates. Over longer times, cavity growth can take place by the separable process of vacancy diffusion along grain boundaries, driven by the applied stress and resulting in vacancy condensation to enlarge continually the cavity size. ${ }^{18)}$ These processes may be coupled so that both may make simultaneous contributions to cavity growth.

In circumstances where vacancy diffusion makes the largest contribution, the rate of increase $d v / d t$ in cavity volume is given by

$$
d v / d t=\pi D_{g} w \sigma \Omega / k T
$$

There is experimental support for Eq. (15) in only a limited number of cases. ${ }^{19}$ ) It holds for some pure metals and when cavities are not continually being created it follows that

$$
t_{R} \propto 1 / \sigma
$$

This is in marked contrast with the Monkman-Grant relationship and may be considered to represent an extreme limit of behaviour. It does however reflect one of the reasons for the observed decrease in $m$ in Eq. (12) with decrease in stress in most circumstances, as materials generally tend towards such behaviour under creep conditions conducive to long creep life.

Nevertheless, it would be far too optimistic to extrapolate creep life on the basis of $\mathrm{Eq}$. (12) since it is only in rare situations that $m$ does not decrease substantially and may approach a value as low as two. There are several reasons why $m$ does not decrease according to the expectations of the early theory of cavity growth by vacancy diffusion and condensation and three major reasons will be outlined here.

First, the grain boundaries in most creep resistant materials are unable to act as perfect vacancy sources. ${ }^{20)}$ A threshold stress may be required to be overcome before any vacancy emission and, above such a threshold, it may still be a more time consuming event to extract a vacancy from a grain boundary than the time required for its diffusion from its source to the cavity.

The second reason ${ }^{21)}$ is that cavities may be constrained from growing at the expected rate because of the insufficient rate of deformation of the material as a whole. This feature becomes important when there is an uneven distribution of cavities in the material so that most of the imposed load is taken by the uncavitated regions of the material.

The third reason ${ }^{22}$ is that cavities may be continually created during deformation in a way that is related both to the strain and to the applied stress. This nucleation problem still presents many puzzling features which will be considered later.

Despite these latter complications in time to fracture evaluations, it must be emphasised that the greatest progress has been made in the understanding of cavity growth, when compared with the more limited understanding of other factors that relate to creep fracture. ${ }^{23)}$

The influence of cavity shape has also been included in several analyses ${ }^{24}$ with the rate of increase in the radius of a disc shaped cavity $d r / d t$ on the boundary plane given by

$$
d r / d t=D_{s} b \sigma^{3} \Omega / 2 \gamma^{2} k T
$$

where, $b$ : the boundary width

$D_{s}$ : the surface diffusion coefficient.

Such a formula holds when the rate of surface diffusion is insufficient to permit the cavity to retain a nearly spherical shape.

From all these considerations it is thus concluded that volumetric rates of cavity growth may vary with stress within the extreme limits of a linear relation at low stresses and a power-law relationship approximately similar in its exponent to the creep rate $v s$. stress relationship at high stress. In many instances 
of creep fracture, cavity growth laws are near the centre of this wide range. There is much evidence to show that when the growth rate stress relationship is nearly linear then the principal stresses are rate controlling. ${ }^{25}$ When the power law growth relationship tends to follow the creep law, then cavity growth is determined by the deviatoric stress. ${ }^{26)}$

\subsection{Cavity Nucleation}

Cavity nucleation continues to pose the most severe problems in the assessment of creep fracture. ${ }^{23)}$ In many instances there is evidence that grain boundary particles provide nuclei but most results and observations do not suggest a clear correspondence between the numbers of such particles believed initially to be present and the number of cavities which are seen eventually to form. Attempts have been made to list a hierarchy of particles in terms of their effectiveness as cavity nuclei but in most circumstances it seems that nucleation is influenced by additional factors.

Thus it appears likely that the creation of cavities is dependent on grain boundary orientation and on sliding and, in consequence, is influenced by strain and by the stress level imposed.

Many theories of nucleation have been put forward but without any of them being fully supported by experimental results. The majority of experiments suggest a nearly linear relationship between the number of cavities and the strain, at least in the early stages of crecp. ${ }^{27)}$ The influence of stress on nucleation remains unclear. It seems likely that both tensile and shear stress components will both play a part in the nucleation process.

At constant temperature, the number of cavities $\mathcal{N}$ may be written

$$
\mathcal{N}=f_{1}(\sigma, \varepsilon)
$$

where the function $f_{1}$ may approximately be taken to have the form

$$
\mathcal{N}=\propto \sigma^{p} \varepsilon
$$

where $p$ has been found to have values ranging between $2<p<8$. No exponent has been attached to $\varepsilon$, in Eq. (19), though cases have been reported where an exponent less than unity might be appropriate. This feature might then be attributed to an eventual saturation level of nuclei.

It is generally accepted that both nucleation and growth must be considered in any interpretation of creep fracture and this has been related to concepts of creep damage.

\section{Creep Damage}

The existence of individual cavities in leading to creep fracture at low stresses is usually evident through observations of fracture surfaces by scanning electron microscopy. ${ }^{22)}$

Their formation and growth can be inferred from observations of polished and etched surfaces (with ion bombardment etching particularly successful) at various stages of creep life. Much evidence supports the view that the final fracture occurs when the level of cavitation builds up to a given fraction of the crosssectional area. This implies that stress concentrations are of only minor importance and useful developments in understanding have followed from the early concepts of Kachanov ${ }^{28)}$ and Robotnov ${ }^{29)}$ of creep damage. These concepts have first represented a phenomenological approach using a single state variable $\omega$ which can be defined in physical terms as an integral of cavity nucleation and growth rates. Many factors can be taken into account using this approach, but in the simplest terms and at constant temperature, the strain rate can be described by

$$
\dot{\varepsilon}=f_{3}(\sigma, \omega)
$$

where the function $f_{3}$ is defined so that Eq. (20) is identical with the steady state creep equation when $\omega=0$ and fracture occurs when $t=t_{R}$ corresponding to a value of $\omega$ equal to unity. The rate of increase of the damage parameter $\dot{\omega}$ prior to fracture can be described by

$$
\dot{\omega}=f_{4}(\sigma, \omega)
$$

and the form of the function $f_{4}$ must now be determined. Assuming a simple form and introducing reference levels $\varepsilon_{0}, \sigma_{0}$, and $\omega_{0}$, we may write

and

$$
\left.\begin{array}{rl}
\dot{\varepsilon} / \dot{\varepsilon}_{0} & =\left(\sigma / \sigma_{0}\right)^{n} /(1-\omega)^{n} \\
\dot{\omega} / \dot{\omega}_{0} & =\left(\sigma / \sigma_{0}\right)^{x} /(1-\omega)^{y}
\end{array}\right\}
$$

from these it may be shown further that

and

$$
\left.\begin{array}{rl}
\omega & =1-\left[1-\left(t / t_{R}\right)\right]^{1 /(y+1)} \\
t_{R} & =1 /(y+1) \stackrel{\circ}{0}_{0}\left(\sigma / \sigma_{0}\right)^{x}
\end{array}\right\}
$$

The formalisation of creep damage in Eqs. (22) and (23) does not depend on the geometrical description of $\omega$ but to provide a scientific basis and improve the realism and accuracy of the relationships, it is important to incorporate into $\omega$ both cavity nucleation and growth. It may be considered ${ }^{30)}$ that at a time $\tau$ the rate of nucleation is $\dot{\mathcal{N}}(\tau)$ so that, in a time interval $d \tau$, the number of new voids formed is $\dot{\mathcal{N}}(\tau) d \tau$. At some later time $t$, the cavities already formed will be growing and the rate of increase in their cross-sectional area at the grain boundaries of

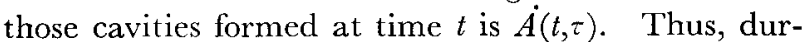
ing the time interval $(t-\tau)$, cavities formed at time $t$ will have a cross sectional given by

$$
A=\dot{\mathcal{N}} d \tau \int_{\tau}^{t} \dot{A}(t, \tau) d t
$$

Now $\omega=A / A_{0}$ where $A_{0}$ is the initial area of crosssection and so taking $\omega=1$ at the time $t=t_{R}$, then $A=A_{0}$ and so

$$
A_{0}=\int_{\tau=0}^{\tau=t_{R}} \dot{N}(\tau) d \tau \int_{t=\tau}^{t=t_{R}} \dot{A}(t, \tau) d t
$$

At the present time there is only limited data from which the approach characterised by Eq. (25) can be used, but the requirements for predictive methods on this basis now become more clear. 


\section{Conclusions}

Prediction of the extent of creep and the occurrence of creep fracture requires the simplest formulation from which extrapolations can be made with confidence. Many equations have been proposed for such purposes and their usefulness has been examined in terms of their probable accuracy and the extent to which they can be understood on a scientific basis.

It is clear that a number of individual mechanisms account for a wide range of phenomena and relationships. The mechanisms can often be separately identified but they may be coupled in ways that provide a basis for some of the empirical relationships derived from experimental data. There is much scope for physical modelling in the further development of prediction methods.

\section{REFERENCES}

1) J. H. Gittus: Creep, Viscoelasticity and Creep Fracture, Applied Sci. Pub., London, (1975).

2) H. Kraus: Creep Analysis, John Wiley \& Sons, New York, (1980).

3) R. K. Penny and D. L. Marriott: Design for Creep, McGraw Hill, London, (1971).

4) J. Cadek: Materials Science Monographs, 48 Elsevier, Amsterdam, (1988).

5) H. Oikawa: Creep and Fracture of Engineering Materials and Structures, ed. by B Wilshire and D.R.J. Owen, Pineridge Press, Swansea, UK, (1981), 113.

6) R. W. Evans and B. Wilshire: Creep of Metals and Alloys, Inst. Met., London, (1985), 204.

7) U. F. Kocks, A. S. Argon and M. F. Ashby: Progress in Materials Science, Vol. 19, ed. by B. Chalmers, Pergamon Press, Oxford, (1975), 1.

8) M. F. Ashby: Acta metall., 20 (1972), 887.
9) E. Artz, M. F. Ashby and R. A. Verrall: Acta metall., 31 (1983), 377.

10) F.R.N. Nabarro: Report on Conference on the Strength of Solids, Phys. Soc., London, (1948), 75.

11) R. L. Coble: J. Appl. Phys., 34 (1963), 1679.

12) M. F. Ashby: Scr. metall., 3 (1969), 837.

13) G. W. Greenwood: Phil. Mag., A51 (1985), 537.

14) B. Burton and G. W. Greenwood: Mater. Sci. Technol., 2 (1986), 1029.

15) R. W. Evans, I. Beden and B. Wilshire: Proc. 2nd Int. Conf. on Creep and Fracture of Engineering Materials and Structures, ed. by B. Wilshire and D.R.J. Owen, Pineridge Press, Swansea, UK, (1984), 1277.

16) F. C. Monkman and N.J. Grant: Proc. ASTM, 56 (1956), 593.

17) J. A. Williams: Acta metall., 15 (1967), 1559.

18) D. Hull and D. E. Rimmer: Phil. Mag., 4 (1959), 673.

19) M. D. Hanna and G. W. Greenwood: Acta metall., 30 (1982), 833.

20) J. E. Harris, R. B. Jones, G. W. Greenwood and M. J. Ward: J. Aus. Inst. Met., 14 (1969), 154.

21) B. F. Dyson: Can. Met. Quart., 18 (1979), 31.

22) B. J. Cane and G. W. Greenwood: Met. Sci., 9 (1975), 55.

23) H. E. Evans: Mechanisms of Creep Fracture, Elsevier Sci. Pub., Barking, (1984).

24) T. J. Chung, K. I. Kagawa, J. R. Rice and L. B. Sills: Acta metall., 27 (1979), 265.

25) G. W. Greenwood: Phil. Mag., 43 (1981), 281.

26) B. F. Dyson: Creep and Fracture of Engineering Materials and Structures, ed. by B. Wilshire and D.R.J. Owen, Pineridge Press, Swansea, UK, (1981), 235.

27) W. Beere: Cavities and Cracks in Creep and Fatigue, ed. by John Gittus, Applied Sci. Pub., Barking, UK, (1981), 25.

28) L. M. Kachanov: Izv. Akad. Nauk SSSR, Metally, (1958), No. 8, 26.

29) Y. N. Rabotnov: Creep Problems in Structural Members, North-Holland Publ. Co., Amsterdam, (1969).

30) R. Raj and M. F. Ashby: Acta metall., 23 (1975), 653. 